\title{
Trend in rifampicin-, multidrug- and extensively drug-resistant tuberculosis in Italy, 2009-2016
}

\author{
To the Editor:
}

Tuberculosis (TB) caused by strains of Mycobacterium tuberculosis that are rifampicin-resistant (RR), multidrug-resistant (MDR) (strains resistant to at least isoniazid and rifampicin) or extensively drug-resistant (XDR) (MDR strains resistant to any fluoroquinolone (FQ) and to at least one second-line injectable drug (SLID): kanamycin, capreomycin or amikacin) is a major threat to TB control globally. All three groups require treatment with second-line drugs (SLDs) [1]. We previously reported that MDR- and XDR-TB in Italy occurred mostly in foreign-born persons (FBPs) [2, 3], but no major information on RR, MDR or XDR trends in FBPs and Italian-born persons (IBPs) were shown. Here, we documented these trends from 2009 to 2016 (figure 1).

In our country, the surveillance of drug-resistant $\mathrm{TB}$ is coordinated by the World Health Organization (WHO) Supranational Reference Laboratory (SRL) in Rome collaborating with the Italian Multicentre Study on Resistance to Antituberculosis Drugs (SMIRA), a laboratory network presently comprised of 42 laboratories in 18 out of 20 regions, and with the WHO SRL in Milan. In 2015, the SMIRA network, which is periodically examined by first-line drug and SLD proficiency testing exercises [2, 4], covered $69.3 \%$ of nationwide notified cases [5] and contributed the majority of cases included in annual reports of European Centre for Disease Prevention and Control/WHO.

In 2009-2016, a total of 15985 M. tuberculosis strains from TB patients with known nationality (mean \pm SD $2004 \pm 121$ strains per year) were isolated. Strains from patients with unknown nationality $(\mathrm{n}=3083)$ were not included in the study. Figure 1a shows that most MDR/RR strains were isolated from FBPs, and that MDR and RR (any resistance to rifampicin, whether monoresistance, polyresistance, MDR or XDR) rates in FBPs decreased in parallel with those of FBPs+IBPs. MDR rates in FBPs+IBPs decreased with a mean annual change of $-3.8 \%$, in keeping with $-2.8 \%$ and $-3.8 \%$ MDR-TB decline reported for the European region in 2004-2013 [6] and 2008-2012 [7], respectively. In IBPs, the RR rates decreased while MDR rates stabilised at $0.6 \pm 0.1 \%$.

Starting from 2011, the SMIRA laboratories determined SLD susceptibility, and from 2011 to 2016, reported data on 375 MDR strains isolated from 12240 TB cases, including 300 MDR cases from 6845 FBPs and 75 MDR cases from 5395 IBPs. The trends showed that resistance of 300 FBP strains to both FQs and SLIDs (XDR strains) was stable (10.8土1.6\%) (figure 1b). Despite data fluctuations, trends were quite stable also for resistance to FQs $(22.6 \pm 5 \%)$ and SLIDs $(27.4 \pm 4 \%)$, and for susceptibility to SLIDs+FQs $(48.1 \pm 7.3 \%)$. As to the 75 IBP strains, the corresponding values were $1.9 \pm 1.6 \%, 4.1 \pm 3 \%$, $5.5 \pm 2.9 \%$ and $7.6 \pm 5.3 \%$, respectively, indicating that resistances to SLIDs and/or FQs were much lower in IBPs than in FBPs.

Out of 15985 M. tuberculosis strains examined, 7248 (45.3\%) were isolated from IBPs and 8737 (54.7\%) from FBPs who had arrived in Italy from 107 countries, including 65 countries with non-MDR and 42 with MDR strains (734 and 8003 strains, respectively). The 8003 FBP strains were stratified into six geographical areas by continent (Europe, Africa, Asia and the Americas) and continent regions (figure 1ce). Due to known differences in MDR-TB in Europe and Africa [2, 5, 8], Europe was subdivided in Eastern Europe (EE) and the former Soviet Union (FSU), and Africa into north Africa (NA) and

@ERSpublications

In Italy, rifampicin-resistant and MDR-TB were high in foreign-born persons, but decreased from 2009 to 2016 http://ow.ly/MrIy30jNjFj

Cite this article as: Mustazzolu A, Borroni E, Cirillo DM, et al. Trend in rifampicin-, multidrug- and extensively drug-resistant tuberculosis in Italy, 2009-2016. Eur Respir J 2018; 52: 1800070 [https://doi.org/ 10.1183/13993003.00070-2018]. 

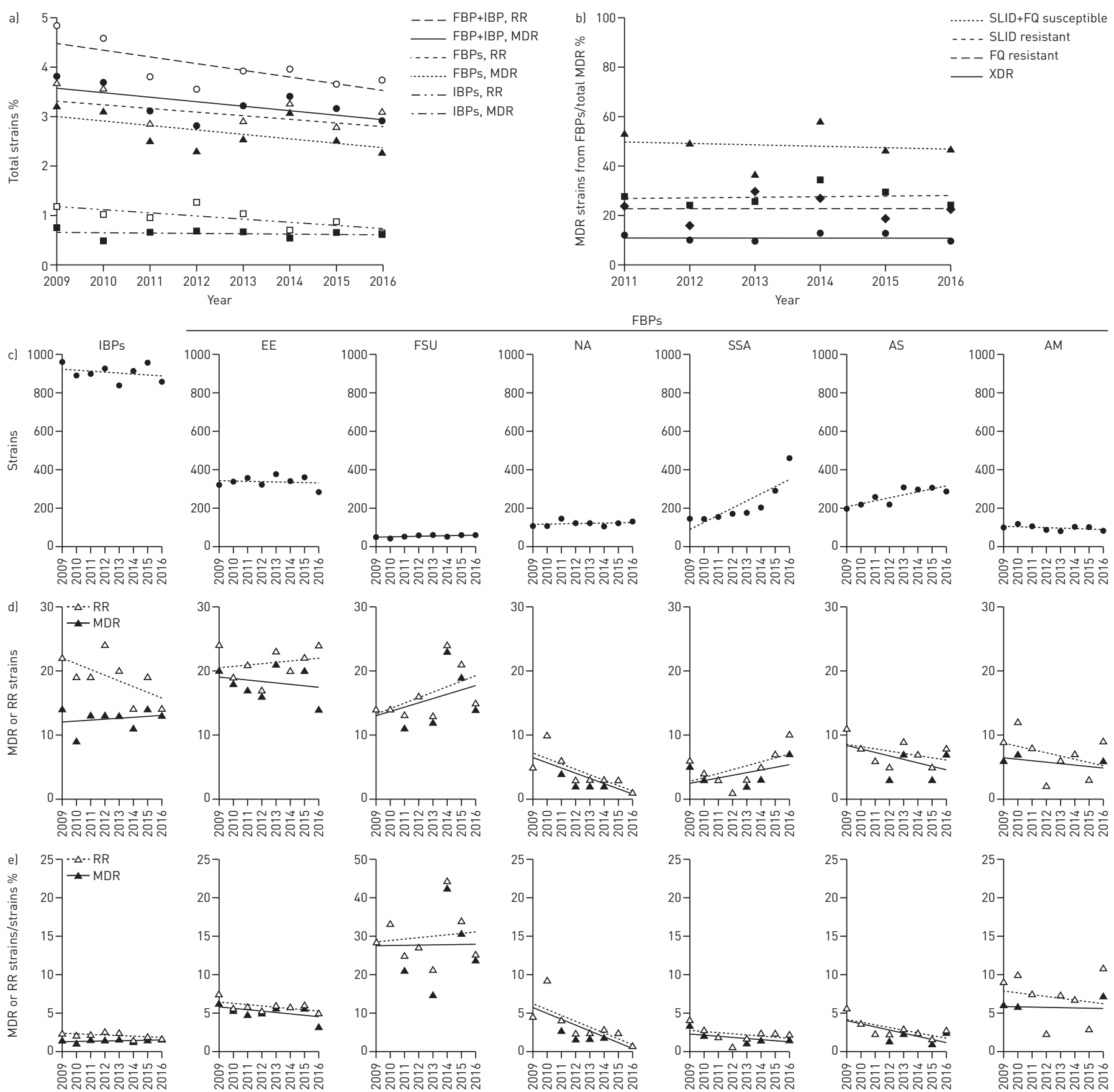

FIGURE 1 Trends in rifampicin-resistant (RR), multidrug-resistant (MDR) and extensively drug-resistant tuberculosis in foreign-born persons (FBPs) and Italian-born persons (IBPs). a) RR and MDR rates in FBP+IBP, FBPs and IBPs. b) Susceptibility and/or resistance of MDR strains from FBPs to second-line injectable drugs (SLIDs) and fluoroquinolones (FQs). c) Number of strains isolated from IBPs and FBPs. d) Number of RR and MDR strains from IBPs and FBPs. e) RR and MDR rates in IBPs and FBPs. EE: Eastern Europe; FSU: former Soviet Union; NA: north Africa; SSA: sub-Saharan Africa; AS: Asia; AM: the Americas.

sub-Saharan Africa (SSA). MDR strains were also isolated from FBPs who had arrived from Asia (AS) and the Americas (AM).

The numbers of strains from the top country of each FBP area were as follows. EE: Romania, $n=2237$; NA: Morocco, $n=782$; AS: Pakistan, $n=590$; SSA: Senegal, $n=470$; AM: Peru, $n=402$; FSU: Ukraine, $n=215$. The RR and MDR rates showed different patterns when compared with the absolute number of strains: Ukraine, $30.2 \%$ and 28.4\%; Peru, $8.2 \%$ and 7.0\%; Romania, $6.4 \%$ and $5.9 \%$; Morocco, $3.5 \%$ and $3.1 \%$; Pakistan, 2.2\% and 1.7\%; and Senegal $1.1 \%$ and $1.1 \%$, respectively. These values are consistent with 
MDR/RR rates in new cases in their native countries: Ukraine, 27\%; Peru, 6.3\%; Romania, 2.8\%; Morocco, 1\%; Pakistan, 4.2\%; and Senegal, 0.9\% [1].

The number of FBPs in Italy and worldwide has continued to grow in recent years [9-11]; thus, we investigated the trends of RR- and MDR-TB in IBPs and FBPs by stratifying data in seven areas (IBPs and six areas for FBPs), and indicating tendency by linear trend lines. The numbers of strains are reported in figure $1 \mathrm{c}$ while the numbers of RR- and MDR-strains are shown in figure $1 \mathrm{~d}$. Finally, the RR and MDR rates are reported in figure $1 \mathrm{e}$.

Figure 1c shows that amongst IBPs and FBPs, the FSU countries had the lowest numbers of strains. All FBP areas showed stable trends with the exception of SSA and AS, in which numbers increased by 3.1 and 1.5 times, respectively, from 2009 to 2016. The SSA increase was mostly due to migrants arriving from Senegal, Nigeria, Somalia, Gambia, Eritrea and Ivory Coast; the AS increase to migrants from Pakistan, India and the Philippines. As to the numbers of RR and MDR strains (figure $1 \mathrm{~d}$ ), in IBPs the RR decreased while MDR stabilised at $12.5 \pm 1.7$ strains per year. In FBPs, the numbers of MDR strains from EE, NA, AS and AM decreased while those from FSU and SSA increased.

Figure 1e showed that, in general, MDR rates decreased in all areas, with the exception of IBPs and FSU, which displayed the lowest and highest values, respectively, and stabilised at $1.4 \pm 0.2 \%$ and $27.7 \pm 8.4 \%$, respectively. About $90 \%$ of FSU strains were isolated from FBPs coming from Ukraine, Moldova and Russia, in keeping with our previous study [2]. As for SSA, despite the rise in numbers of strains (figure 1c) and of MDR and RR strains (figure 1d) from 2009 to 2016, MDR and RR rates were low and decreasing, in keeping with a recent meta-analysis on MDR-TB in SSA [8].

Overall, all trends in MDR rates were lower than those of RR rates and decreased, with exception for the IBP, FSU and AM groups, which remained quite constant. MDR and RR rates showed declining parallel trends for EE, NA and SSA, while RR tended to converge to MDR from 2009 to 2016 for IBPs and AM, and to slightly diverge for FSU and AS. These trend differences between RR and MDR rates are in keeping with the knowledge that the proportion of RR strains susceptible to isoniazid vary in different areas of the world, i.e. RR-TB is not always a good proxy for diagnosis of MDR-TB [1, 12-14]. Thus, in order to obtain a correct diagnosis of MDR-TB, detection of rifampicin resistance by rapid molecular assays (e.g. Xpert MTB/RIF) should be followed by rapid detection of isoniazid resistance.

In summary, this study showed that from 2009 to 2016, the MDR/RR-TB rates decreased in FBPs who arrived in Italy from several countries, with exception of the FSU, in which they continue to have very high levels. This is in keeping with the knowledge that of the 10 countries in the world with the highest rates of MDR-TB, nine are in the FSU, where rates have not decreased in recent years $[1,5]$. Some limitations of this study are that it did not cover all notifications and that it was not possible to distinguish new from previously treated cases due to difficulties in obtaining reliable history of prior treatment. Implementing an Italian version of the European Respiratory Society (ERS)/WHO Consilium based on the ERS platform may contribute to eliminate TB in our country [15].

Alessandro Mustazzolu ${ }^{1}$, Emanuele Borroni ${ }^{2}$, Daniela Maria Cirillo $^{2}$, Federico Giannoni ${ }^{1}$, Angelo Iacobino ${ }^{1}$, the Italian Multicentre Study on Resistance to Antituberculosis Drugs (SMIRA) ${ }^{3}$ and Lanfranco Fattorini ${ }^{1}$

${ }^{1}$ Dept of Infectious Diseases, Istituto Superiore di Sanità, Rome, Italy. ${ }^{2}$ Emerging Bacterial Pathogens Unit, San Raffaele Scientific Institute, Milan, Italy. ${ }^{3} \mathrm{~A}$ full list of the SMIRA group members and their affiliations can be found in the Acknowledgements section.

Correspondence: Lanfranco Fattorini, Dept of Infectious Diseases, Istituto Superiore di Sanità, Via Regina Elena 299, 00161 Rome, Italy. E-mail: lanfranco.fattorini@iss.it

Received: Jan 122018 | Accepted after revision: April 242018

Acknowledgements: The members of the SMIRA laboratory network are: V. Ghisetti and A. Mondo (Hospital Savoia, Turin); M. Avolio and A. Barbui (Hospital Molinette, Turin); P. Lorenzetti (Hospital of Aosta, Aosta); G. De Renzi and M.G. Chirillo (Hospital of Orbassano, Orbassano); G. Molinari, A. Camaggi and S. Andreoni (Hospital of Novara, Novara); F. Piana (Hospital of Cuneo, Cuneo); A. Marchese, P. Gritti, G. Icardi and O. Varnier (Hospital San Martino, Genoa); E. Mazzola and G. Gesu (Hospital Niguarda, Milan); P. Cichero (Hospital San Raffaele, Milan); A. Lombardi (Hospital Sacco, Milan); E. Libanori, P. Viggiani and S. De Lorenzo (Hospital of Sondalo, Sondalo); G. Pinsi (Spedali Civili, Brescia); P. Marone, V. Monzillo and D. Barbarini (Hospital San Matteo, Pavia); C. Farina and M. Arosio (Hospital Giovanni XXIII, Bergamo); M. Peracchi and R. Manganelli (Hospital of Padova, Padua); C. Fabris, M. Di Santolo and M. Busetti (Hospital of Trieste, Trieste); C. Scarparo and A. Sartor (Hospital of Udine, Udine); C. Pedrotti and I. Caola (Hospital of Trento, Trent); E. Frizzera (Hospital of Bolzano, Bolzano); P. Dal Monte (Hospital San Orsola-Malpighi, Bologna); P. Pietrosemoli, M. Pecorari, A. Fabio and A. la Regina (Hospital of Modena, Modena); M. Matteucci (Area Vasta Romagna, Pievesestina, Cesena); C. Piersimoni (Hospital of Ancona, Ancona); A. Bartolesi, R. Mannino, T. Simonetti and E. Tortoli (Hospital Careggi, Florence); L. Rindi (University of Pisa, Pisa); A. Mencacci, E. Cenci, E. Luciano and R. Mazzolla, (Hospital of Perugia, Perugia); I. Sanguigni, G. Parisi, P. Chiaradonna and A.M. Altieri (H. San Camillo-Forlanini, Rome); S. D’Arezzo, A. Mazzarelli, A. Di Caro and E. Bordi (Hospital Spallanzani, 
Rome); M. Sali, G. Delogu and M. Sanguinetti (Hospital Gemelli, Rome); C. Russo, L. Coltella and A. Ciocco (Hospital Bambino Gesù, Rome); M. Meledandri (Hospital San Filippo Neri, Rome); A. Gambi and G. Tomei (Hospital of Chieti, Chieti); M. Conte, G. Santoro and A. del Giudice (Hospital dei Colli, Naples); N. Nuzzolese, E. Vitullo, A. Sinno and L. Buono (Hospital of Matera, Matera); D. Costa (Hospital of Bari, Bari); A. Grimaldi (Hospital of Triggiano, Bari);

A. Di Taranto and R. De Nittis (Hospital of Foggia, Foggia); G. Palumbo (Hospital of Lecce, Lecce); S. Dodaro,

C. Giraldi and P. Cavalcanti (Hospital of Cosenza, Cosenza); S. Nisticò and L. Vinci (Hospital of Lamezia Terme,

Lamezia Terme); C. Di Naso (Hospital Garibaldi Centro, Catania); C. Bonura, C.M. Maida and C. Mammina (Hospital Giaccone of Palermo, Palermo); and G.S. Podda and R. Caddeu (Hospital of Cagliari, Cagliari).

Support statement: This study was supported by the CCM Project of the Ministry of Health, in the framework of the DPCM 03.03.2017 (GU 109, 12.05.2017) on the Surveillance systems and Registry of mortality, cancer and other pathologies. Funding information for this article has been deposited with the Crossref Funder Registry.

Conflict of interest: None declared.

\section{References}

1 World Health Organization. Global Tuberculosis Report 2017. WHO/HTM/TB/2017.23. Geneva, WHO, 2017.

2 Fattorini L, Mustazzolu A, Borroni E, et al. Tuberculosis in migrants from 106 countries to Italy, 2008-2014. Eur Respir J 2016; 47: 1273-1276.

3 Fattorini L, Mustazzolu A, Piccaro G, et al. Drug-resistant tuberculosis among foreign-born persons in Italy. Eur Respir J 2012; 40: 497-500.

4 Fattorini L, Migliori GB, Cassone A, et al. Proficiency testing of first- and second-line anti-tuberculosis drugs in Italy. Eur Respir J 2012; 39: 1263-1266.

5 European Centre for Disease Prevention and Control. Surveillance Report: Tuberculosis Surveillance and Monitoring in Europe - 2017. https://ecdc.europa.eu/sites/portal/files/documents/ecdc-tuberculosis-surveillancemonitoring-Europe-2017-WEB.pdf

6 European Centre for Disease Prevention and Control. Surveillance Report: Tuberculosis Surveillance and Monitoring in Europe - 2015. http://ecdc.europa.eu/en/publications/Publications/tuberculosis-surveillancemonitoring-Europe-2015.pdf

7 European Centre for Disease Prevention and Control. Surveillance Report: Tuberculosis Surveillance and Monitoring in Europe - 2014. https:/ecdc.europa.eu/sites/portal/files/media/en/publications/Publications/ tuberculosis-surveillance-monitoring-Europe-2014.pdf

8 Musa BM, Adamu AL, Galadanci NA, et al. Trends in prevalence of multi drug resistant tuberculosis in sub-Saharan Africa: a systematic review and meta-analysis. PLoS One 2017; 12: e0185105.

9 ISTAT. GeoDemo. http://demo.istat.it/

10 Ingrosso L, Vescio F, Giuliani M, et al. Risk factors for tuberculosis in foreign-born people (FBP) in Italy: a systematic review and meta-analysis. PLoS One 2014; 9: e94728.

11 Matteelli A, Centis R, Sulis G, et al. Crossborder travel and multidrugresistant tuberculosis (MDRTB) in Europe. Travel Med Infect Dis 2016; 14: 588-590.

12 Kurbatova EV, Cavanaugh JS, Shah NS, et al. Rifampicin-resistant Mycobacterium tuberculosis: susceptibility to isoniazid and other anti-tuberculosis drugs. Int J Tuberc Lung Dis 2012; 16: 355-357.

13 Nasiri MJ, Zamani S, Pormohammad A, et al. The reliability of rifampicin resistance as a proxy for multidrug-resistant tuberculosis: a systematic review of studies from Iran. Eur J Clin Microbiol Infect Dis 2018; 37: 9-14.

14 World Health Organization. Companion handbook to the WHO guidelines for the programmatic management of drug-resistant tuberculosis. WHO/HTM/TB/2014.11. Geneva, WHO, 2014.

15 Blasi F, Matteelli A, Sotgiu G, et al. Moving towards tuberculosis elimination: a call for action from Italy and a possible model for other low tuberculosis incidence countries. Eur Respir J 2017; 49: 1602242. 